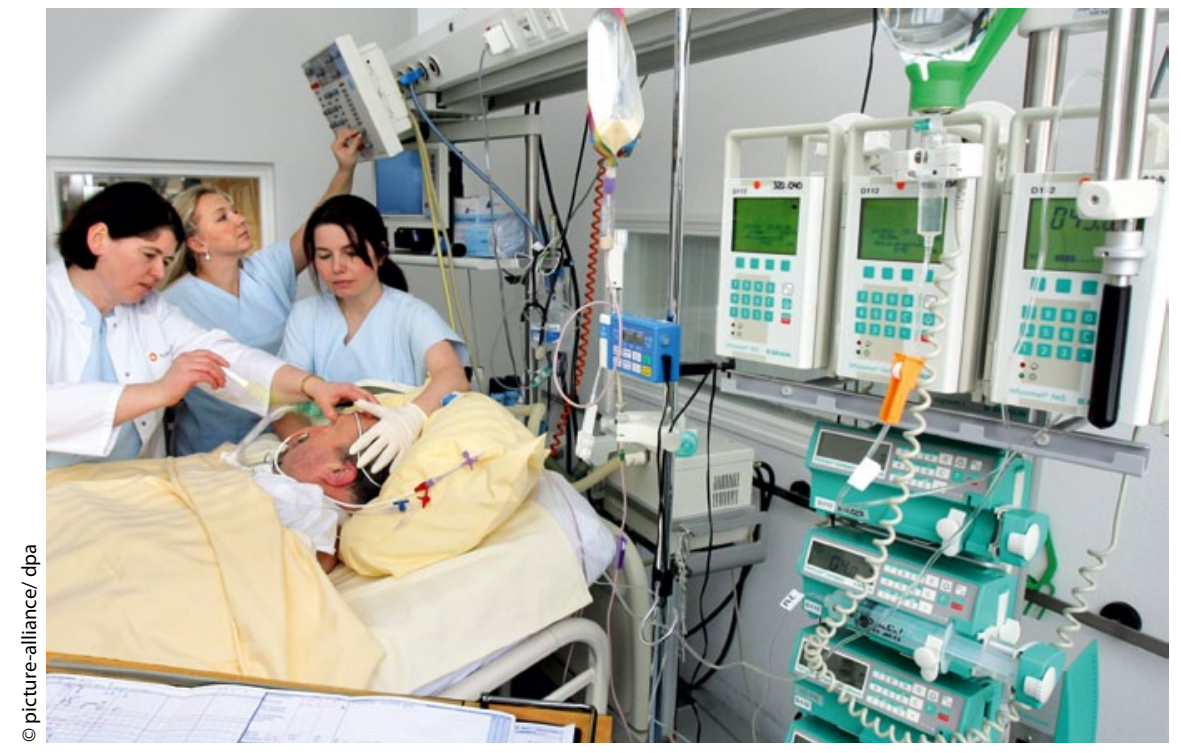

In einer großen, doppelblinden Studie versagte Citicolin gegenüber Placebo in 24 Stunden nach den ersten Schlaganfallsymptomen. Citilocin ist in der Behandlung des mittelbis hochgradigen Schlaganfalls also nicht wirksam.

\title{
Citicolin beim akuten Schlaganfall doch nicht wirksam
}

In einigen Ländern ist Citicolin zur Behandlung des akuten Schlaganfalls zugelassen. In Deutschland ist es als CDP-Cholin als "diätetisches Lebensmittel für besondere medizinische Zwecke" (ergänzende bilanzierte Diät; Handelsname: (eraxon ${ }^{\oplus}$ ) erhältlich. In Analysen aus früheren Jahren gab es Hinweise, dass die Gabe dieser Substanz zu einem besseren Outcome beim Schlaganfall führt. Die folgende Studie konnte diese These nicht bestätigen.

Z um ersten Mal wurde in diesem $\mathrm{Zu}$ sammenhang eine große randomisierte multizentrische Studie durchgeführt, an der auch deutsche Zentren beteiligt waren (sogenannte ICTUS Studie). Die Studie war doppelblind angelegt, es wurden Patienten mit einem mittel- bis hochgradigen Schlaganfall eingeschlossen. Sie erhielten entweder Citicolin oder Placebo innerhalb von 24 Stunden nach den ersten Schlaganfallsymptomen (1.000 mg alle 12 Stunden intravenös während der ersten drei Tage, dann über sechs Wochen $2 \times 500 \mathrm{mg}$ Tabletten).

Als primärer Endpunkt war die Erholung nach 90 Tagen definiert. Darin gingen der National Institutes of Health Stroke Scale, der modified Rankin Score und der Barthel Index mit ein. Außerdem wurden symptomatische intrakranielle Blutungen, neurologische Verschlechterung und Mortalität erfasst. Insgesamt wurden 2.298 Patienten bis zum Oktober 2011 eingeschlossen. 1.148 Patienten erhielten Citicolin und 1.150 erhielten Pla- cebo. Die Studie wurde bei der dritten Interimanalyse auf der Basis von 2.078 Patienten gestoppt. Die globale Erholung vom Schlaganfall war ungefähr in beiden Gruppen gleich (OR 1,03; 95 \% CI 0,86$1,25 ; \mathrm{p}=0,364)$. Auch hinsichtlich weiterer Wirksamkeits- und Sicherheitsparameter wurden keinerlei Unterschiede zwischen beiden Gruppen gefunden. Zusammenfassend zeigt die ICTUS-Studie, dass die Gabe von Citilocin in der Behandlung des mittel- bis hochgradigen Schlaganfalls nicht wirksam ist.

Kommentar: Diese Studie zeigt einmal mehr, dass Substanzen, die in kleineren Studien, anekdotischen Berichten und dann auch in Metaanalysen eine gewisse Wirksamkeit für die Erholung von einem Schlaganfall gezeigt haben, in großen, placebokontrollierten Studien versagen. Die Gabe von Citiclin in der ICTUS-Studie zeigte durchaus, dass in der Tat diese Substanz die Erholung von einem Schlaganfall nicht unterstützt. Sie sollte daher nicht empfohlen werden, zumal sie von den Patienten selber bezahlt werden muss.

Prof. Dr. med. Dr. phil. Stefan Evers

Dávalos A, Alvarez-Sabín J, Castillo J, DíezTejedor E, Ferro J, Martínez-Vila E, Serena J, Segura T, Cruz VT, Masjuan J, Cobo E, Secades $\mathrm{JJ}$; for the International Citicoline Trial on acUte Stroke (ICTUS) trial investigators. Citicoline in the treatment of acute ischaemic stroke: an international, randomised, multicentre, placebo-controlled study (ICTUS trial). Lancet 2012 [Epub ahead of print]

\section{Sagt das Spot-Sign das Outcome nach intrazerebralen Blutungen vorher?}

Intrazerebrale Blutungen haben gegenüber ischämischen Infarkten eine deutlich schlechtere Prognose. Deswegen ist es um so wichtiger, ihr Outcome vorherzusagen. Klassischerweise werden zur Prognoseabschätzung die Größe der Blutung, der Wert auf der Glasgow-Coma-Scale, intraventrikuläre Blutungen und das Lebensalter herangezogen. Die vorliegende Studie zeigte, dass auch der Nachweis von Spot-Signs mit einem schlechteren Outcome assoziiert ist.

$\mathrm{D}$ as Spot-Sign entsteht, wenn man ein CT mit Kontrastmittel durchführt; es zeigt einen Austritt von Kontrastmittel aus rupturierten kleinen Gefäßen an. Dies könnte ein Hinweis darauf sein, dass es weiter nachblutet und die Prognose der Blutung insgesamt schlecht ist. Die vorliegende kanadische Studie ist eine prospektive multizentrische Beobachtungsstudie, in die Patienten mit einer Hirnblutung von 